\title{
A collaborative method to survey and store urban components
}

\author{
Qingyun Tang ${ }^{1,2 *}$, Guiwen Lan ${ }^{1,2}$, Donglin Fan ${ }^{1,2}$, Yongliang Du ${ }^{1,2}$ \\ ${ }^{1}$ College of Geomatics and Geoinformation, Guilin University of Technology, Guilin, Guangxi, 541006, China \\ ${ }^{2}$ Guangxi Key Laboratory of Spatial Information and Geomatics, Guilin, Guangxi, 541006, China
}

\begin{abstract}
Urban components are the important part of the city, and the rapid and efficient survey of urban components is a key requirement of urban digitization. In this paper, a method of the collaborative survey and storage of urban components is proposed. The national standard code for urban components survey is optimized to accelerate the survey and storage of urban components, and the time cost of error discovery is shorten by using AutoCAD to check the spatial location and employing a VBA macro programme to search for the attribute data errors. At the same time, a collaborative processing flow of components data is constructed through ArcPy to further speed up the storage of urban components. The experiment of urban components survey in Wanxiu District of Wuzhou City shows that this method can effectively reduce the complexity of urban component data storage procedure and the error rate. Compared with the traditional method, the proposed method is about 2 times more efficient to input the urban component data into the database.
\end{abstract}

\section{Introduction}

Urban components are the important part of the city, and are also an important data source of the so-called "digital city" ${ }^{11}$. Effective city management requires us to have a better way to obtain the information such as quantity, category, location, attributes, distribution, and so on, of the urban components. However, the survey of urban components is often time-consuming and need very great effort to collect their information one by one, and it is quite easy to make mistakes when processing the data and inputting them into the database. Therefore, it is necessary to establish an efficient method to facilitate the survey of urban components.

Although some existing basic software, such as AutoCAD, ArcGIS, SuperMap, and MapGIS, are able to process urban components data, but they mostly focus on the basic editing function of spatial data, and do not deal with the storage of urban components. Therefore, these software are not able to support efficient entry of urban components data ${ }^{[2]}$. In the conventional technical process of urban components survey and storage, field workers rely on basic map data to obtain the geographical location of urban components and record their attribute information in the field. And then the quality of the field data is checked by the office operators, and the data for each field survey is entered into the database one by one. If there is an error, field workers need to go to the site for quality inspection and rechecking attribute data ${ }^{[3]}$. The way of conventional components data storage is easy to cause errors, which are mainly reflected in the following aspects: the first is the spatial position error. This is mainly caused by the measurement equipment and human factors. For example, the street trees are not on the same horizontal line, and the distance between the rain grate and the edge of the road is large. There are obvious logic errors in these spatial positions. The second is the attribute data error. This is mainly caused by human error. The error is that setting the street lamp category to the manhole category, having obvious factual errors for height and width of the tree, and mismatching the photos of the interest points, etc. The third is the attribute data omission. This is mainly caused by that some required components attributes are not collected. For example, the photos of the interest points, the heights and radiuses of the trees and the numbers of the parking lot, etc., are not collected.

As for the deficiency of traditional urban component survey, this paper optimize the unified code rules for the fast survey of urban components based on the national standard code. And the VBA macro programme, AutoCAD and ArcPy are used to complete the batch data processing and implement the storage and management of the urban components data on ArcGIS.

\section{Collaborative survey and storage technology of urban components}

In order to improve the efficiency of quality survey and storage, project managers often divide a task into several parts. When managing multi-task parallel storage, they

*Corresponding author's e-mail: 649811715@qq.com 
need to understand the processing procedure of all workers in real time, so as to adjust the workload at any time according to the progress. The workers should also be clear about the scope of their own operations, and simultaneously be aware of the operation progress of other members, so as to coordinate with other workers.

In this paper, combined with the relevant norms of urban components survey, the survey data processing process is reorganized to construct the collaborative storage mode of three standards (spatial location check, attribute information check and component quality check) and two processing (pre-processing and verification processing). As shown in Figure 1, this process mainly involves two steps: one is to expand the code of urban components survey, so as to parse into attribute information in the later stage. The other is to use ArcPy plugin to implement data storage, automatic layering, automatic symbolization and batch drawing, etc. The collaborative survey and storage process of urban components constructed in this paper can realize the collaborative processing, transparent inspection and fast storage of the data, etc., which greatly improves the survey and storage efficiency of urban components.

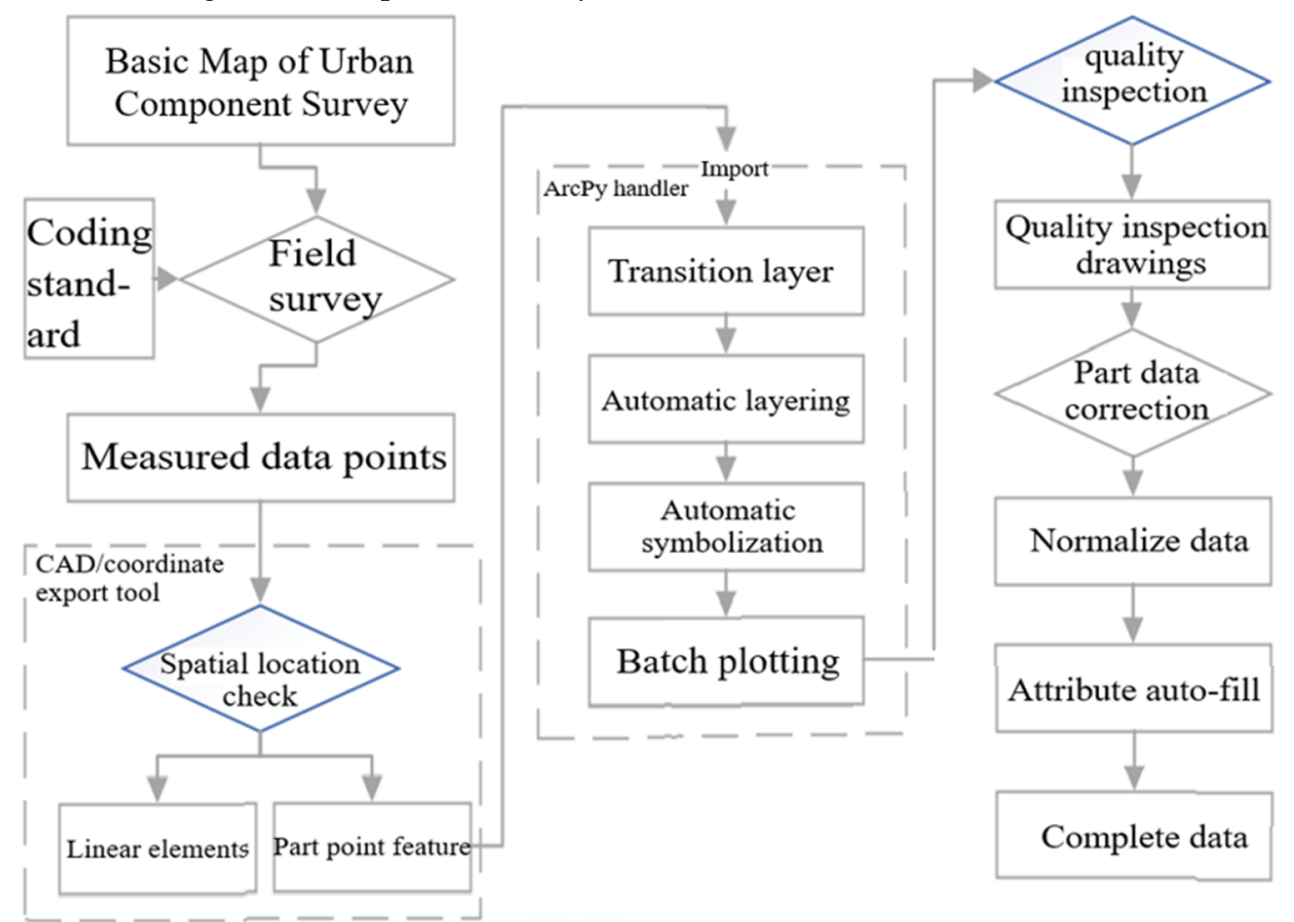

Figure 1. Flow chart of fast collaborative survey and storage of urban components.

\subsection{Extension of survey codes for urban components}

According to the classification code of national urban components survey, a type of urban components is generally represented by four digits, which can only identify different types of components, but cannot distinguish the attributes of the same type of components. To parse the code into the types and attributes of the components, expanding and optimizing the original classification code is essential ${ }^{[4]}$. Therefore, seven to nine digits are used to code the urban components. The first digit code is the usage status of the components, indicating that the status of the components is intact/broken/lost/occupied, etc.; the second to fifth digit codes are the national standard classification code of the components, representing the types of the urban components; the sixth to ninth digit codes are the extended attribute code, representing the material, quantity and other attributes of urban components. For example, the code of a communication manhole cover is
10107112, and its the first digit "1" means intact, the second to fifth digits "0107" signifies the type of communication manhole cover, the sixth digit "1" denotes cast iron, the seventh digit " 1 " indicates that the manhole cover belong to the mobile company, and the eighth digit " 2 " implies that there are two communication manhole covers at this location. For another example, the code of a tree is 104020802 , and its the first digit " 1 " means the tree is in good condition, the second to fifth digits "0402" signifies the type of street tree, and the sixth to seventh digits " 08 " implies the height of the tree is 8 meters, and the eighth to ninth digits " 02 " indicates the radius of the tree crown is 2 meters. If the code of components needs to be updated, the code can be added from the last digit of the original code while the original code remains unchanged. Thus, the code with the corresponding attribute is expanded according to the actual needs, which can greatly facilitate the internal workers to quickly parse the code and get the component attributes, avoid the input of component attributes one by one, and improve the storage efficiency of urban components survey data. The coding method is shown in Figure 2. 


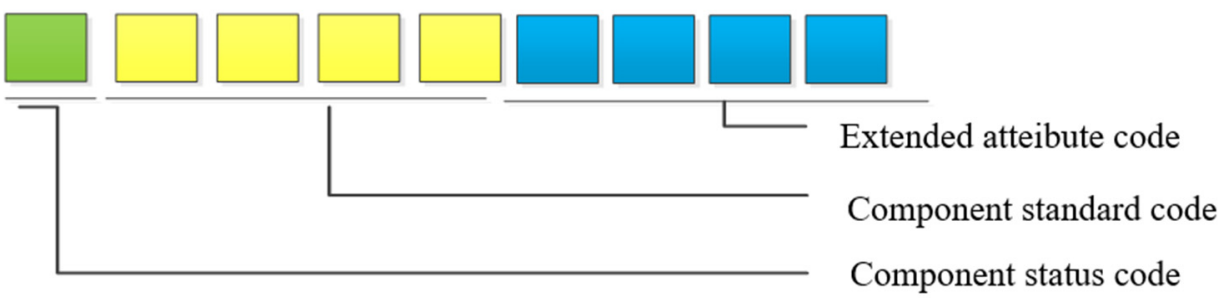

Figure 2. Classification coding schematic diagram.

\subsection{Collaborative processing of urban components survey data.}

In this paper, an ArcPy plugin for ArcGIS is used to modify data, symbolize data, and edit data, and so on. A variety of logic checks, data batch modification and entry can be carried out through this plugin, which can maximize the efficiency of the collaboration of all the users of the ArcPy program.

For an urban component, each mandatory attribute should be checked to make sure whether it is integral, if not, it should be modified according the integrity rules. For example, as some attributes may have a default value, and these attributes will be set to default value when some users do not enter their actual values for the sake of convenience, then it will cause errors and data integrity problem, which make a integrity check necessary. The rules of checking the required attribute information are shown in Table 1. After using the VBA programme to pre-process and check the data, the data is added, deleted and modified according to the feedback information of the program, and then the data is exported for importing into ArcGIS for collaborative processing.

Table 1. Checklist of necessary attribute information.

\begin{tabular}{|c|c|c|c|}
\hline $\begin{array}{l}\text { Serial } \\
\text { No. }\end{array}$ & Items subject to checks & Check description & If required \\
\hline 1 & Code integrity check & $\begin{array}{l}\text { Check if the number of code digits is greater } \\
\text { than } 5\end{array}$ & Yes \\
\hline 2 & $\begin{array}{l}\text { Components condition } \\
\text { check }\end{array}$ & $\begin{array}{c}\text { Check if the first digit of the code is any } \\
\text { value in }[0,1,2] .\end{array}$ & Yes \\
\hline 3 & Components photo check & $\begin{array}{l}\text { Check if street name plates, place name } \\
\text { plates, etc. are missing }\end{array}$ & Yes \\
\hline 4 & $\begin{array}{l}\text { Compliance with the } \\
\text { material of the manhole } \\
\text { cover category }\end{array}$ & $\begin{array}{l}\text { The sixth digit (in the } 7 \text { th digit when there is } \\
1 \text { extra manhole cover) }\end{array}$ & Yes \\
\hline$\ldots$ & $\ldots$ & & \\
\hline
\end{tabular}

During the collaborative processing, the ArcPy plugin for ArcGIS is employed to check the data for errors, automate the data hierarchy, and perform batch symbolisation. Before components data being partitioned for producing a diagram through a self-defined grid, using the rules of the Table 1 to mark the missing items for prompting the quality inspection personnel is necessary, so as to increase the efficiency of quality inspection and simultaneously simplify the work of the internal processing. To get results data that met certain survey accuracy, the ArcPy program is adopted to merge regularised data according to the grid number. The specific method of the collaborative processing is depicted in Figure 3. 


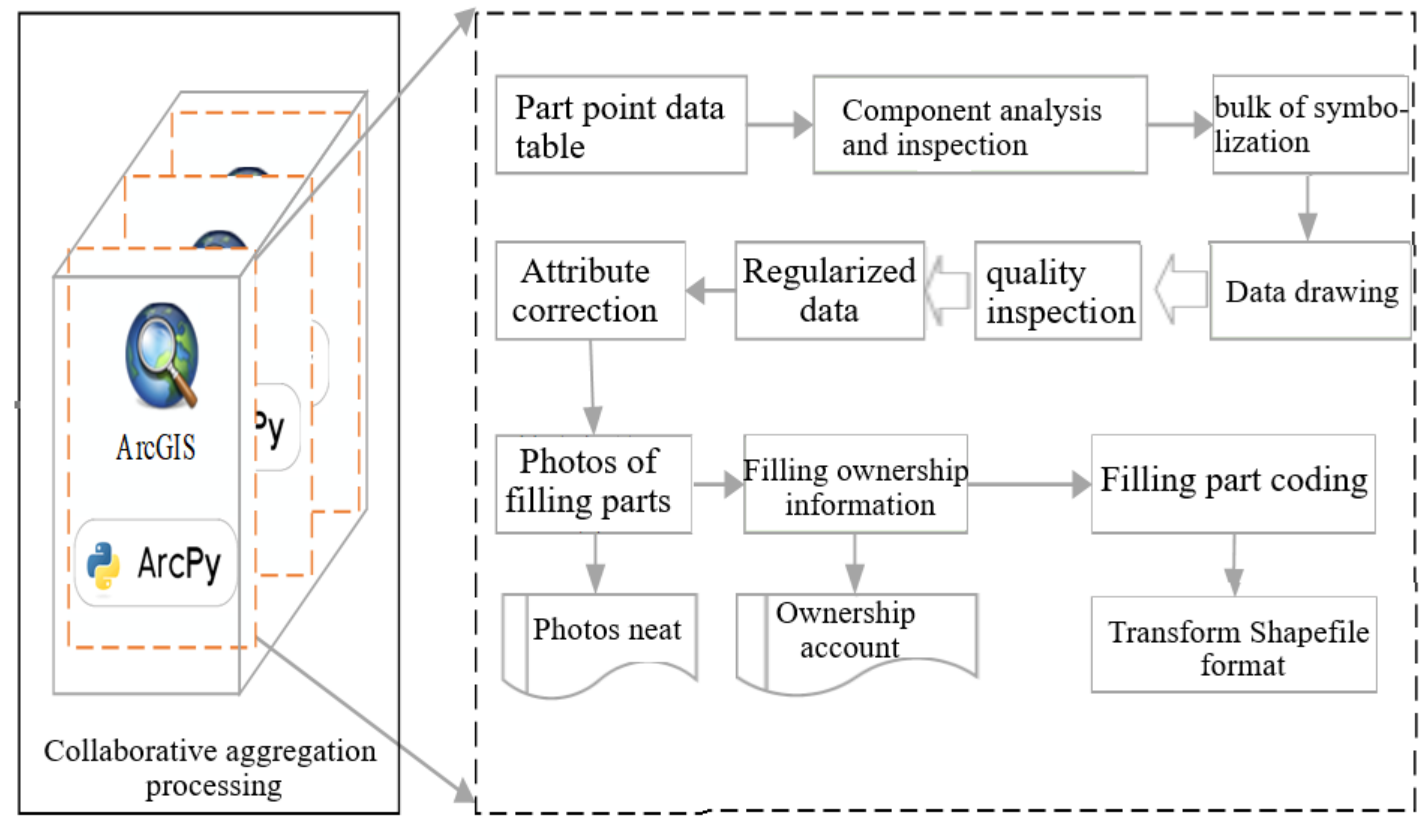

Figure 3. The collaborative process of storing urban components data.

Firstly, processing components data. Using the ArcPy programme to maintain the mapping from the full attribute field to each components attribute field for the purpose of automating the hierarchy of the components data. Then, after completing spatial position inspection, the components data is imported into Excel for parsing the code into the attribute information by using VBA macro programme. If the photos of some components are missing or their names cannot be matched to the components points because of misnaming, the corresponding components points is counted so that the field quality inspectors can take the corresponding photos later. Finally, the component symbol library is made by using the component symbol standard established by the state, and the batch symbolization is carried out according to the relationship between the symbol and the component type.

Secondly, producing map. A diagram is produced for modifying the error data of the components according to the row number and column number when implementing the data quality inspection. Attribute correction is to convert the codes into corresponding text, while performing several logic checks to correct unreasonable attributes.

Thirdly, organizing the photos. There are two cases when organizing the photos: In the first case, the quality inspection photos is renamed and transferred to the target path according to the components category and photo coding rules; In the second case, each subtype of the components need to be photographed, and the reorganized according to the small category and named according to the convention naming rules.

Finally, filling in the ownership information. Founded on the components ownership account information, an ownership table is created and the components code is filled to meet the requirements for populating required fields.

\section{Case study and analysis}

According to the above technology, the rapid storage technology of urban components survey of the internal and field collaborative operation is be implemented by using VBA program and ArcPy script. This paper takes the survey of the components in Wanxiu District of Wuzhou City as an experiment. In the same area, two groups of the same number of people are selected to carry out the survey and quality inspection respectively through the traditional method and the method proposed in this paper. And then the missing rate, error rate and time cost of survey and storage are counted. The urban components of the Wanxiu District of Wuzhou City are divided into 6 categories and 123 sub-categories. 706 Las points are collected, and 496 urban components were collected in the experimental area of the Wanxiu District with a road section of 3.5 kilometers.

Table 2. Distribution of field staff.

\begin{tabular}{cccc}
\hline measurement method & $\begin{array}{c}\text { Number of field } \\
\text { workers/person }\end{array}$ & equipment & Field work time/day \\
\hline Collaborative survey program & 2 & 1 RTK/1 total station & 1.8 \\
Traditional technology & 2 & 1 RTK/1 total station & 4 \\
\end{tabular}


The traditional method and the collaborative method are used to survey and store the components respectively. The comparison results of components input efficiency are shown in Table 4, and the comparison results of components input and omissions are shown in Table 5. It can be seen from the table that the traditional component data storage is carried out in a timely manner after the end of the field work every day, the component attributes of each field survey must be input one by one, and the working hours of this collaborative survey program is 2 times faster than the traditional technology. The distribution of field staff is shown in Table 2, and the comparison of internal processing treatment plans is shown in Table 3. The efficiency and time of the rapid survey and storage of the collaborative method are significantly better than the traditional method by the statistics. It not only realizes the seamless combination of urban component data information and attribute information, but also improves the accuracy of attribute input on the basis of improving the efficiency of attribute input. After verification of experimental data, this method is 2.14 times faster than the traditional method.

Table 3. Comparison of internal processing treatment plans.

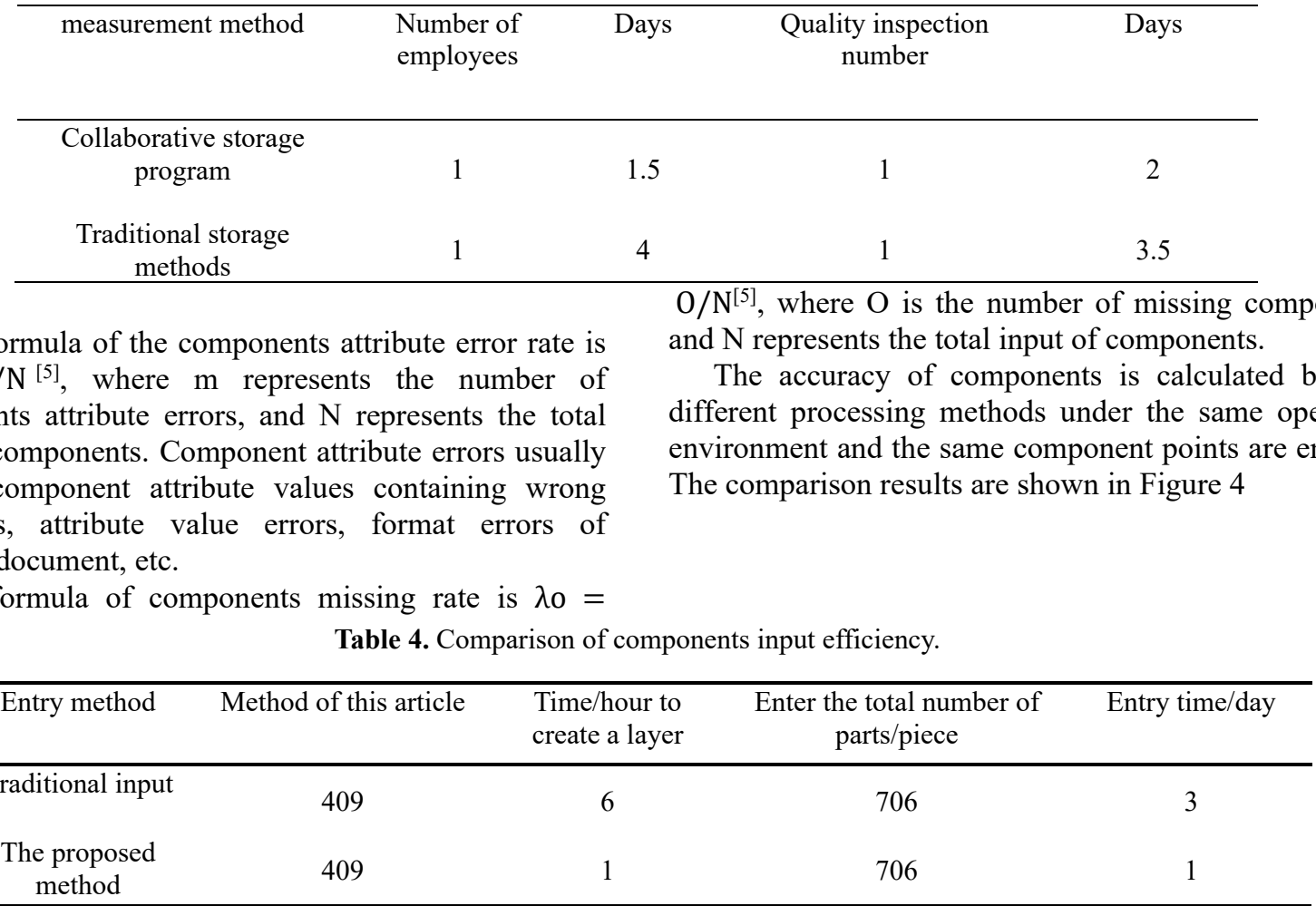

The formula of the components attribute error rate is $\lambda \mathrm{e}=\mathrm{m} / \mathrm{N}^{[5]}$, where $\mathrm{m}$ represents the number of components attribute errors, and $\mathrm{N}$ represents the total input of components. Component attribute errors usually include component attribute values containing wrong characters, attribute value errors, format errors of outcome document, etc.

The formula of components missing rate is $\lambda_{0}=$
$\mathrm{O} / \mathrm{N}^{[5]}$, where $\mathrm{O}$ is the number of missing components $\mathrm{N}$ represents the total input of components.

The accuracy of components is calculated by two environment and the same component points are entered. The comparison results are shown in Figure 4

Table 5. Comparison of components errors and omissions.

\begin{tabular}{ccccc}
\hline Entry method & $\begin{array}{c}\text { Total number of } \\
\text { missing } \\
\text { parts/piece }\end{array}$ & $\begin{array}{c}\text { Components } \\
\text { missing rate\% }\end{array}$ & $\begin{array}{c}\text { Total number of part } \\
\text { attribute errors/piece }\end{array}$ & $\begin{array}{c}\text { Total number of part } \\
\text { attribute } \\
\text { errors/piece } \\
\lambda \mathrm{e} \%\end{array}$ \\
\hline Traditional input & 97 & 1.1 & 102 & 1.17 \\
$\begin{array}{c}\text { The proposed } \\
\text { method }\end{array}$ & 23 & 0.2 & 16 & 0.28 \\
\hline
\end{tabular}

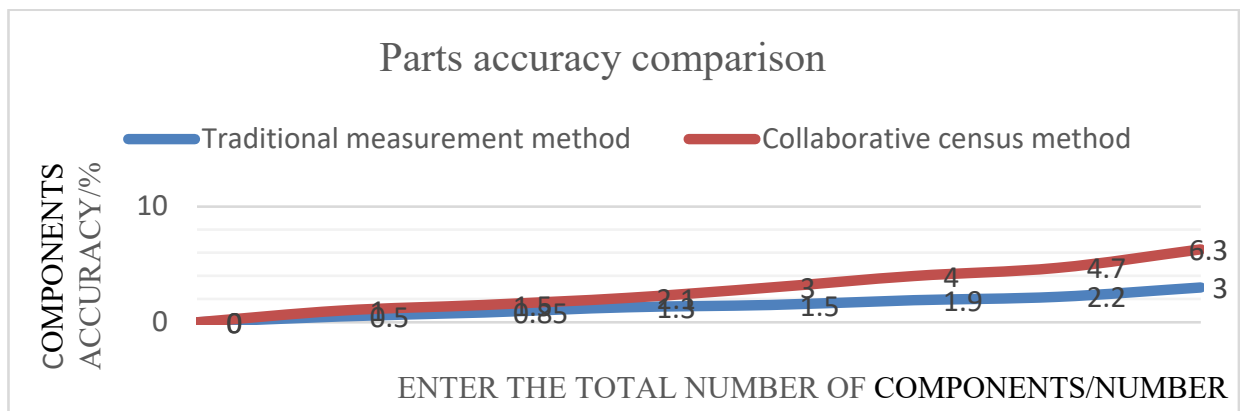

Figure 4. Comparison of components accuracy. 


\section{Conclusion}

In this paper, a method of the collaborative survey and storage of urban components is proposed. The national standard code for urban components survey is optimized to accelerate the survey of urban components, and the AutoCAD is used to check the spatial location and the VBA macro programme is employed to search for the attribute data errors. At the same time, the plugin developed by ArcPy is used to collaboratively process the components data. And this method is validated in the project of urban components survey in Wanxiu District of Wuzhou City. Compared with the traditional method, this method is about 2 times more efficient to survey and store the urban component data. As this method optimizes the work flow, and realizes the online scheduling and cooperation of data processing and quality control, it greatly improves the working efficiency and promotes the unified data management during the urban component survey project. Although the application of the proposed method has proved.

Although this paper attempts to explore this aspect and achieved some results, the method has yet to be further improved in the practical application. Owing to the limitations of the project background, the survey scope is not large in this paper. As a next step, a larger survey scope is needed for verification to optimize the method in this paper.

\section{References}

1. Gao, W., Tian Y.H., Wang J. (2018) Digital retina: revolutionizing camera systems for the smart city. Scientia Sinica (Informationis), 48: 1076-1082.

2. Duan, L., Chandrasekhar, V., Jie, C., Jie, L., \& Wen, G. (2015) Overview of the MPEG-CDVS standard. IEEE Transactions on Image Processing, 25: 323-332.

3. Tan, Y.H. (2019) Research on the construction and standard database of surveying and mapping geographic information in Guangdong province. Journal of Geomatics, 44: 27-28+106.

4. Xu, H., Liu, Y., Tang, X., Liu, Z., Badawi, R., \& Liu, H. (2009). Research on models of digital city geo-information sharing platform. Proceedings of SPIE-The International Society for Optical Engineering, 7492: 74924B-74924B-7.

5. Yan, Z.J., Liu, R.F., Hou, H.L. (2016) Urban components collection and rapid storage technology for mobile measurement system. Surveying and Spatial Geographic Information, 39: 63-65+68. 